\title{
Do Periarticular Joint Injections Present an Increase in Infection Risk? - CORRIGENDUM
}

The title of the article by Seidelman et $\mathrm{al}^{1}$ was incorrect in the final published version. The original title should have read "Injections" instead of "Infections." This has been updated in the original published article. The authors apologize for this error.

\section{REFERENCE}

1. Seidelman J, Baker AW, Anderson DJ, Sexton DJ, Lewis SS. Do Periarticular Joint Injections Present an Increase in Infection Risk? Infection Control Hosp Epidemiol 2018;39:890-891. 